\title{
Comparative Study On Different Features And Classifiers For Emboli Signal Identification
}

\author{
Dzati Athiar Ramli, Najah Ghazali, Haryati Jaafar
}

\begin{abstract}
Occurrence of embolism from patients who suffer from carotid artery stenosis may bring to the onset of stroke if it became severe. In clinical practice, Doppler ultrasound technique is commonly used to detect the emboli in the cerebral circulation. Instead of depending on human observer as a gold standard to detect the emboli, this study proposes an automated embolic identification system based on ultrasound signal analysis. Experimental studies on 1,400 samples from five independent data sets are employed in this study. Two feature extraction methods based on spectral feature i.e. Linear Prediction Coefficient (LPC) and statistical features i.e. combination of Measured Embolus-to-Blood Ratio (MEBR), Peak Embolus-to-Blood Ratio (PEBR), entropy, standard deviation and maximum peak are used to extract the signal. Subsequently, four classifiers based on nearest neighbor approach i.e. $k$ Nearest Neighbor $(k N N)$, Fuzzy k-Nearest neighbor (FkNN), k Nearest Centroid Neighbor (kNCN), and Fuzzy-Based k-Nearest Centroid Neighbor (FkNCN) are used to evaluate the performance of the identification system. The experimental results show that FkNCN with statistical feature outperforms the other classifiers with the performance of $\mathbf{9 2 . 4 5} \pm \mathbf{2 . 1 2} \%$ is achieved.
\end{abstract}

Keywords - Embolus detection; Transcranial Doppler Ultrasound; Feature extraction; Classification.

\section{Introduction}

Stroke is a disease due to the present of tiny particles called embolus which, travel in the arteries that connected toward and within the brain. A large amount of embolus may block the carotid system and this affects the amount of blood flowing toward the brain which leads to stroke event. The main symptoms of stroke can be detected through transient ischemic attack (TIA) or mini stroke that may occur within 24 hours, feeling weakness on one side of the body, clumsiness of the hands or fingers and in more serious cases which are related to inability to walk and to move the arms or facial muscles. Currently, stroke disease is the third cause of death and leads to high possibility of disability in Malaysia. Until now, stroke patients are hard to be fully recovered as it give permanent impairment of nerves system and some medication is only available for recovery of the mini stroke [1].

Dzati Athiar Ramli

Universiti Sains Malaysia, Malaysia

Najah Ghazali

Universiti Sains Malaysia,Malaysia

Haryati Jaafar

Universiti Sains Malaysia, Malaysia
Following that, current research on stroke has focused on the study of embolus detection at the high risk location that commonly present in the middle cerebral artery in cerebral system [2]. The ability to observe the embolization rate and lesion in the identified location may improve the pre-clinical treatments to decrease the risk of stroke event among patients.

Currently, clinical practice in observing the occurrence of embolus in blood vessels under human skulls is done by using Transcranial Doppler (TCD) ultrasound machine [3]. By determining the frequency of embolus event, TCD can identify the conditions of the artery in which the blockage may affect the amount of blood flowing toward the brain [4]. Nevertheless, the detection of emboli based on TCD is still relying on human observer as a gold standard. As analyzing significant information of emboli event from the TCD machine is quite tedious and time-consuming job, thus an automatic identification system is greatly needed for detection of embolus occurrence.

Previous studies on the embolus detection based on machine learning methods have been reported by many researchers. Recently a relatively new classification method, binary decision tree [5], Artificial Neural Network (ANN), K-Nearest Neighbors Rule (KNNR)[6][7] and Support Vector Machines (SVM) [8] have been proposed. The results shown that the accuracy performance to detect artefact and emboli composition is greater than $80 \%$. However, there is no comparative study have been done among the classifiers performances. In this study, a comparative study on features selection and types of classifiers for emboli identification system based on ultrasound signal analysis is experimented. The multifrequency datasets are considered, following the previous technique that relies on the ultrasound beam pattern at frequencies range of 1.0 to $3 \mathrm{MHz}$ [9][10]. The developed system consists of three important procedures i.e. data collection, feature extraction and classification.

The signal data were obtained by using a transcranial Doppler (TCD) system simulator. Subsequently, the signal data were extracted with the spectral feature i.e. Linear Prediction Coefficient (LPC) and statistical features i.e. the combination of Measured Embolus-to-Blood Ratio (MEBR), Peak Embolus-to-Blood Ratio (PEBR), entropy, standard deviation and maximum peak as the feature extraction [4][7]. In order to classify the embolus in the pattern matching process, various classifiers from the Nearest Neighbor (NN) family were used including $\mathrm{k}$ Nearest Neighbor $(\mathrm{kNN})^{8}$, Fuzzy k-nearest neighbor (FkNN) [9], k nearest centroid neighbor (kNCN) [11], and Fuzzy-Based kNearest Centroid Neighbor (FkNCN) [12].

This paper has been outlined as follows. In Section 2, the methodology of this study including data acquisition, feature extraction and classification are discussed. The experimental 
results are presented in Section 3 and finally, Section 4 provides the conclusion.

\section{Methodology}

\section{A. Data Collection}

The Transcranial Doppler Ultrasound Signal Simulator (version 2.3, Switzerland) was employed for database collection. A transducer was placed on the right and left side of the transtemporal (region) for the patients who were having more than $80 \%$ of symptomatic internal carotid artery stenosis. The sampling frequency was $8 \mathrm{KHz}$. Five independent data sets of patients' group with different probe position and transmitted frequency $(1 \mathrm{MHz}-3 \mathrm{MHz})$ are defined as in Table 1. All groups had the recording time of up to 40 minutes with the Doppler instrument's sample volume depth was placed near the skull between $58 \mathrm{~mm}$ to $62 \mathrm{~mm}$.

When an observer perceived the Doppler sonogram and suspected the emboli event, the permanent storage of the contents of the buffer was activated. As a result, a total of 244 MES signals had been detected. Each signal was selected according to the chirping sound and sonogram image of the velocity profile as shown in Fig. 1. The desired signal was then chosen and randomly cropped with the time error estimation between $5 \mu$ s to $7 \mu$ s within the time delay. The reason of considering the time delay in the targeted signal is to mimic the real situation in which the error in a measurement would occur and unexpected calibration would therefore has to be encountered. The signals of the extracted features were then analyzed offline.

TABLE I. The PARAMETERS For Simulated TIME SERIES FOR ULTRASOUND PROBE

\begin{tabular}{|c|c|c|c|}
\hline Group & Frequency Setup & Probe location & SVL Depth \\
\hline $\mathbf{1}$ & $2 \mathrm{MHz}$ & Right Side MCA & $60 \mathrm{~mm}$ \\
\hline $\mathbf{2}$ & $1.5 \mathrm{MHz}$ & Right Side MCA & $60 \mathrm{~mm}$ \\
\hline $\mathbf{3}$ & $2 \mathrm{MHz}$ & Left Side MCA & $58 \mathrm{~mm}$ \\
\hline $\mathbf{4}$ & $1 \mathrm{MHz}$ & Left Side MCA & $62 \mathrm{~mm}$ \\
\hline $\mathbf{5}$ & $3 \mathrm{MHz}$ & Left Side MCA & $62 \mathrm{~mm}$ \\
\hline
\end{tabular}

*MCA : Middle Cerebral Artery; SVL : Sample Volume Length.

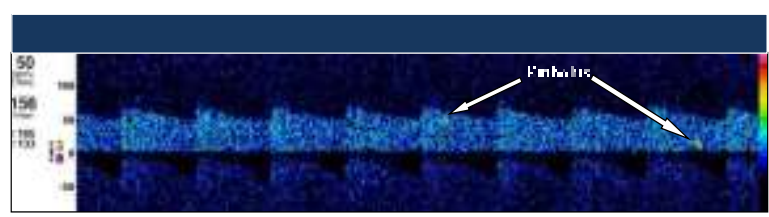

Figure 1. Figure shows the analysis of embolic signals using TCD simulator at $2 \mathrm{MHz}$ transmit frequency and at depth of $50 \mathrm{~mm}$ from the probe placement.

\section{B. Feature Extraction}

In this study, two features from the spectral feature and statistical features were used for the development of the emboli detection. For the spectral feature, the LPC was employed in this study. LPC feature extraction models the process of signal production and is defined as a digital method for encoding an analogue signal in which a particular value is predicted by a linear function of the past values of the signal [13]. The most important aspect of LPC is the linear predictive filter which allows the value of the next sample to be determined by a linear combination of previous samples. In other word, linear prediction filters attempt to predict future values of the input signal based on past signals. LPC analysis is based on the assumption that the relation between the current sample $x(n)$ and first-order linear combination of the previous $\mathrm{p}$ samples given as:

$$
\mathrm{x}(\mathrm{n}) \approx \mathrm{a}_{1} \mathrm{x}(\mathrm{n}-1)+\ldots+\mathrm{\alpha}_{\mathrm{p}} \mathrm{x}(\mathrm{n}-\mathrm{p})
$$

The linear predicted value $\tilde{\mathrm{x}}(\mathrm{n})$ with prediction coefficients, $\sigma_{i}$ for $\mathrm{x}(\mathrm{n})$ is presented as:

$$
\tilde{\mathrm{x}}(\mathrm{n})=\sum_{\mathrm{i}=1}^{\mathrm{p}} \mathrm{a}_{\mathrm{i}} \mathrm{x}(\mathrm{n}-\mathrm{i})
$$

Consequently, LPC cepstrum can be derived through the LPC model. For a time sequence $x(n)$, complex cepstrums $\tilde{\mathrm{c}}(\mathrm{n})$ are represented as below:

$$
\begin{aligned}
& \tilde{\mathrm{c}}(\mathrm{n})=-\alpha_{1} \\
& \tilde{\mathrm{c}}(\mathrm{n})=-\alpha_{\mathrm{n}}-\sum_{\mathrm{m}=1}^{\mathrm{n}-1}\left(1-\frac{\mathrm{m}}{\mathrm{n}}\right) \alpha_{\mathrm{m}} \tilde{\mathrm{c}}_{\mathrm{n}-\mathrm{m}} 1<\mathrm{n} \leq \mathrm{p} \\
& \tilde{\mathrm{c}}(\mathrm{n})=-\alpha_{\mathrm{n}}-\sum_{\mathrm{m}=1}^{\mathrm{p}}\left(1-\frac{\mathrm{m}}{\mathrm{n}}\right) \alpha_{\mathrm{m}} \tilde{\mathrm{c}}_{\mathrm{n}-\mathrm{m}} \mathrm{n}<\mathrm{p}
\end{aligned}
$$

The database of LPC features in this study consists of 1200 set LPC features from the 5 groups and 14 cepstrum coefficients per frame were extracted in this method.

In the meantime, the combination of MEBR, PEBR, entropy, standard deviation and maximum peak were employed for the statistical features. The MEBR is the feature based on the ratios of the embolus to background signal within given short time interval. It can be computed in the time domain [14] and frequency domain [15]. The MEBR measured from the time domain representation is due to the intensity of the signal which can be clearly observed on the detected signal. The increase in the intensity is observed because of the passage of the embolus relative to the background signal. It can be measured by using (6).

$$
\operatorname{MEBR}=10 \log _{10}\left(\frac{I_{\text {emb+blood }}}{I_{\text {blood }}}\right)
$$

where $I_{\text {emb+blood }}$ is the intensity of the embolus and the background signal and $I_{\text {blood }}$ is the intensity of the background signal. The value of intensity is defined as the power of two local maximum of the signal peaks as shown in Fig. 2. Meanwhile, PEBR is computed by replacing the average peak with the maximum peak of the signal in order to measure any significant difference between these two features. The values obtained by the MEBR and PEBR vary depending on the transmitted frequency, length of the attenuation of sample volume and focusing area.

The entropy is used to separate the useful signal from the background noise. The calculation for the entropy can be stated as in (7). 


$$
H(x)=-\sum_{i=1}^{N} p\left(x_{i}\right) \log _{10} p\left(x_{i}\right)
$$

where $x=\left\{x_{1}, x_{2}, \ldots, x_{N}\right\}$ is a set of random signal and $p\left(x_{i}\right)$ is a probability of a random signal $x_{i}$.

The other statistical features such as the standard deviation and maximum peak were also evaluated because of the randomness and measured distances of certain value for each sampling frequency of the signal. The standard deviation as written in (8), measures the fluctuation of the signal from the mean of the signal

$$
\sigma=\frac{1}{N-1}\left[\sum_{i=0}^{N-1} x_{i}^{2}-\frac{1}{N}\left(\sum_{i=0}^{N-1} x_{i}\right)^{2}\right]
$$

where $x_{i}$ is the signal amplitude in the time domain and $N$ is the total number of frequency sampling.

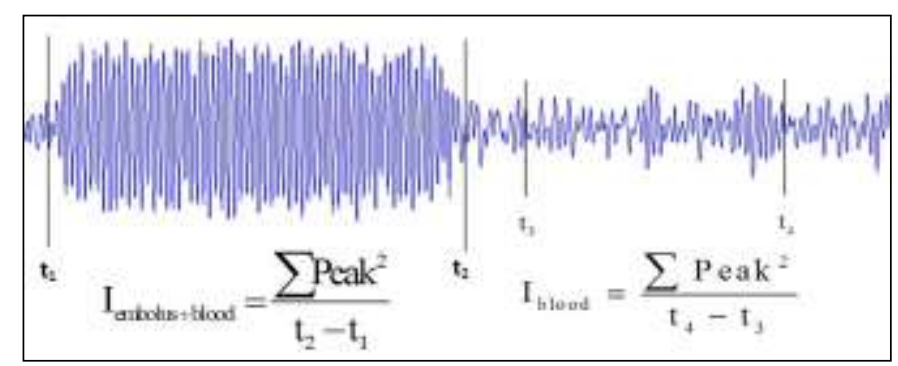

Figure 2. Calculation of intensity obtained from the audio signal.

\section{Classification}

Classification technique in this work represents the technique of identifying emboli from the background signal based on a training set which its membership category is known. In this study, four classifiers i.e. kNN, FkNN, kNCN and FkNCN were investigated to identify embolic signal based on the extracted features.

The kNN has been regularly used in practice since it is simple to be implemented and quite straightforward in the classification process. Moreover, it works fast for small training samples and does not need any prior knowledge about the structure in the data of the training samples [8]. Given a query point $y$, a set of training samples $T=\left\{x_{j}\right\}_{j=1}^{N}$ with the label classes, $c_{1}, c_{2}, \ldots n$, where $N$ is the number of training samples, $x_{j}$ is the training sample and $n$ is the number of class. In order to select the $\mathrm{kNN}$ from the training samples, the distance between the query point and the training samples is determined as written in (9).

$$
d\left(y, x_{j}\right)=\sqrt{\left(y-x_{j}\right)^{T}\left(y-x_{j}\right)}
$$

where $d\left(y, x_{j}\right)$ is the Euclidean distance, $N$ is the number of training samples, $x_{j}$ is the training sample and $y$ is the query point.

In the absence of prior knowledge, most of the $\mathrm{kNN}$ classifiers use the Euclidean distance to measure the distance or similarity between the query point and training samples. The distances are consequently arranged in ascending order and the $\mathrm{kNN}$ is selected based on the training sample that has the smallest distance to the query point. Subsequently, the query point is classified by assigning the class label by the majority voting using the formula in (10).

$$
y=\arg \max \sum_{\left(x_{j}^{N}\right)} w c_{M}
$$

where the weight can be represented as:

$$
w=\left\{\begin{array}{cl}
1 & c_{M}=x_{j}{ }^{N} \\
0 & \text { otherwise }
\end{array}\right.
$$

where $x, y$ are training and testing samples composed of feature $N$, respectively.

On the other hand, the FkNN is an extensive study of $\mathrm{kNN}$ to solve the weighting issues in the kNN. This classifier is assigned a fuzzy membership for the query point rather than assigning the query point to the class label. The fuzzy membership is determined based on the weighted class and is given by (12).

$$
u_{i}(y)=\frac{\sum_{j=1}^{k} u_{i j}\left(1 /\left\|y-x_{j}\right\|^{2 /(m-1)}\right)}{\sum_{j=1}^{k} 1 /\left\|y-x_{j}\right\|^{2 /(m-1)}}
$$

where $i=1,2, \ldots, c, c$ is the number of classes, $u_{i j}$ is the membership degree of training sample, $x_{j}$ ithe one which is selected as the nearest neighbor, $\left\|y-x_{j}\right\|$ is the L-norm distance between the query point $x$ and its nearest neighbor and $m$ is a fuzzy strength parameter which is used to determine how heavily the distance is weighted when calculating the contribution of each neighbor to the fuzzy membership values. The highest fuzzy membership value is considered as a winner for that query point. There are two ways to define $u_{i j}$. One is by using the crisp membership where the training samples assign all of the memberships to their known class and non-memberships to the other class. The other way is by using the constraint fuzzy membership such that when the $\mathrm{k}$ nearest neighbors of each training samples is found ( class is assigned as (13).

$$
u_{i j}\left(x_{k}\right)=\left\{\begin{array}{cc}
0.51+0.49\left(n_{j} / \mathrm{k}\right) & j=i \\
0.49\left(n_{j} / \mathrm{k}\right) & j \neq i
\end{array}\right.
$$

where $n_{j}$ denotes the number of neighbors of $j$ th training samples.

Subsequently, the query point is arranged to the class of with which it has the highest membership value:

$$
c(y)=\arg \max \left(u_{i}(y)\right)
$$

The kNCN is another extensive study of the kNN where this classifier was used a Surrounding Neighborhood (SN) rule to employ the distances and distributions of the training samples before the classification process. According to the $\mathrm{SN}$ rule, the distances of the $\mathrm{k}$ centroid neighbors should be closed to the query point as possible. On the other hand, the distribution of training samples is taken into account such that the $\mathrm{k}$ centroid neighbors are placed symmetrically around the query point. In this classifier, the first nearest centroid neighbor is determined by the shortest distance 
between the query point and training samples using (9). The subsequent nearest centroid neighbors, $x_{i}^{N C N}$ for $i=2, \ldots, \mathrm{k}$ is found by calculating the centroid point, $x_{i j}{ }^{c}$ where the formula of the centroid point is:

$$
x_{i j}{ }^{c}=\frac{\left(\sum_{i=1}^{i-1} x_{i}^{N C N}\right)+x_{j}}{i}
$$

The $i^{\text {th }}$ nearest centroid neighbor can be found by computing the closest distance between the centroid point, $x_{i j}{ }^{c}$ and a query point which is given as:

$$
d\left(y, x_{i j}{ }^{c}\right)=\min \left(\sqrt{\left(y-x_{i j}{ }^{c}\right)^{T}\left(y-x_{i j}{ }^{c}\right)}\right)
$$

The query point is assigned to the class label which is most frequently represented by the $\mathrm{k}$ centroid nearest neighbors using the majority voting in (10).

Finally, the FkNCN employed the surrounding-fuzzy based rule to find the nearest centroid neighbour. This classifier involved both of the centroid neighborhoods and the fuzzy membership. The main objective of the FkNCN was to optimize the performance results while considering the information of samples distribution and fuzzy membership values. Likewise to $\mathrm{kNCN}$, the first centroid neighbor of this classifier is determined by selecting the training sample that was located the nearest to the query point. The first nearest centroid neighbor was set as $x_{1}{ }^{N C N}$. The subsequent nearest centroid neighbors for $i=2, \ldots, \mathrm{k}$ are determined by calculating the centroid position of all the training samples except the training sample that had been selected as the nearest centroid neighbor. The centroid of a set of training samples, $x_{i j}{ }^{c}$ can be defined as in (15).

The $\mathrm{k}$ nearest centroid neighbor was determined by computing the shortest distance between the centroid of a set of a training samples and the query point. It was given as in (17).

$$
x_{r}{ }^{N C N}=\min \left\|\left(y-x_{r j}{ }^{c}\right)\right\|_{L 2}
$$

The process was repeated by determining the centroid between the training samples and previous nearest centroid neighbors. After the $\mathrm{k}$ nearest centroid neighbors had been determined, the next step was to assign a fuzzy membership for the $\mathrm{k}$ nearest centroid neighbors to class labelled as $y$ rather than voting the $\mathrm{k}$ nearest centroid neighbors as a majority. By assigning the fuzzy membership, the FkNCN classifier became more advantageous as it was able to provide the next potential secondary structural class if the first one was wrongly predicted.

This process was done by determining the weight that was inversely proportional to the distance between $x_{r}{ }^{N C N}$ and $y$. The fuzzy membership found was:

$$
u_{r}^{N C N}(y)=\frac{\sum_{i=1}^{k} u_{r i}\left(1 /\left\|y-x_{i}^{N C N}\right\|^{2 /(m-1)}\right)}{\sum_{i=1}^{k} 1 /\left\|y-x_{i}^{N C N}\right\|^{2 /(m-1)}}
$$

where $r=1,2, \ldots, C$, with $C$ as the number of classes and $u_{r i}$ as the membership degree of $\mathrm{k}$ nearest centroid neighbors.

The value of the fuzzy strength parameter was set at 2 . This was because the fuzzy membership values were proportional to the inverse square of the distance. When it was set at 2, it led to an optimal result in the classification process. In the FkNCN, the constraint fuzzy membership is firstly used to ensure heavier weight is assigned to the training samples in its own class while lighter one was assigned to the other class. However, when the value $u_{r i}$ in constraint membership was infinity, the crisp membership led to a better classification accuracy. Thus, $u_{r i}$ is determined by using the combination of the constraint and crisp fuzzy memberships.

After calculating all memberships for a query point, it was assigned to the class of the highest membership value as shown in (19).

$$
C(y)=\arg \max \left(u_{r}^{N C N}(y)\right)
$$

where $n_{j}$ denotes the number of neighbors of $j$ th training samples.

\section{Experimental Results}

The developed embolic signal identification system has been implemented in Matlab R2009 (b) and tested in Intel Core i7, 2.1GHz CPU, 2G RAM and the Windows 8 operating system. In this experiment, the value of $\mathrm{k}$ was set to 5 for the kNN, FkNN, kNCN and FkNCN classifiers. The experiments were evaluated through $\mathrm{K}$-fold cross-validation method where the value of $\mathrm{K}$ was set to 10 . As ten-fold cross-validation was set, 24 samples were chosen as the training samples, while the remaining samples were used for the testing purpose in this database. The advantage of this method is that all of the test sets are independent and the reliability and accuracy of the results can be improved. The results were performed by employing the CA rate.

Table 2 summarizes results of embolus classification by using spectral features. It was also noticeable that the FkNN and $\mathrm{kNCN}$ performed well than $\mathrm{kNN}$ in all group. The FkNN was also comparable with the $\mathrm{kNCN}$ in terms of CA rate with the differences of less than $2 \%$. However, it was found that the $\mathrm{FkNCN}$ performed the best CA rates in Group 5 with $10.51 \%, 8.29 \%, 4.49 \%$ and $0.04 \%$ better than the ones produced by the $\mathrm{kNN}, \mathrm{FkNN}, \mathrm{kNCN}$, respectively. This advantage occurred due to the combination of fuzzy and centroid in the searching stage. In addition, the FkNCN obtained the best CA rate on each condition with more than $78 \%$ was achieved.

TABLE II. ACCURACY RESULTS OF EMBOLUS CLASSIFICATION BY USING SPECTRAL FEATURES

\begin{tabular}{|c|c|c|c|c|c|}
\hline Classifier & Group 1 & Group 2 & Group 3 & Group 4 & Group 5 \\
\hline kNN & 67.33 & 80.00 & 72.80 & 84.11 & 80.00 \\
$(\%)$ & \pm 5.93 & \pm 1.41 & \pm 3.81 & \pm 2.43 & \pm 3.78 \\
\hline FkNN & 73.77 & 84.00 & 75.20 & 88.74 & 82.22 \\
$(\%)$ & \pm 4.77 & \pm 1.58 & \pm 4.33 & \pm 2.70 & \pm 5.87 \\
\hline kNCN & 72.22 & 82.40 & 77.11 & 88.67 & 86.02 \\
$(\%)$ & \pm 2.41 & \pm 1.14 & \pm 4.73 & \pm 3.21 & \pm 4.62 \\
\hline FkNCN & 78.44 & 86.40 & 78.67 & 89.60 & 90.51 \\
$(\%)$ & \pm 4.98 & \pm 2.07 & \pm 2.41 & \pm 1.14 & \pm 5.33 \\
\hline
\end{tabular}


The summary of the classification results by using statistical features are provided in Table 3. From this table, it was observed that all the classifiers provide satisfactory results of correct classification among groups $(>80 \%)$. The results also showed that the statistical features yield noticeably higher classification accuracy compared to spectral feature for most of the groups. For instance, the absolute improvements in classification accuracy of kNN for Group 1 and FkNCN for Group 3 were 85.02 $\pm 2.07 \%$ and $90.16 \pm 2.24 \%$, respectively. In addition, it was found that the proposed FkNCN was consistently outperformed the other classifiers in terms of the $\mathrm{CA}$ rate.

TABLE III. ACCURACY RESULTS OF EMBOLUS CLASSIFICATION BY USING STATISTICAL FEATURES

\begin{tabular}{|c|c|c|c|c|c|}
\hline Classifier & Group 1 & Group 2 & Group 3 & Group 4 & Group 5 \\
\hline $\mathrm{kNN}$ & 85.02 & 85.00 & 81.87 & 80.69 & 81.38 \\
$(\%)$ & \pm 2.07 & \pm 4.42 & \pm 2.41 & \pm 3.11 & \pm 1.23 \\
\hline FkNN & 86.93 & 88.08 & 86.51 & 85.32 & 84.31 \\
$(\%)$ & \pm 5.81 & \pm 2.34 & \pm 4.55 & \pm 3.23 & \pm 3.31 \\
\hline $\mathrm{kNCN}$ & 87.64 & 87.46 & 87.02 & 86.57 & 84.74 \\
$(\%)$ & \pm 3.42 & \pm 5.24 & \pm 5.33 & \pm 4.12 & \pm 1.44 \\
\hline FkNCN & 88.87 & 92.45 & 90.16 & 91.33 & 89.04 \\
$(\%)$ & \pm 3.01 & \pm 2.12 & \pm 2.24 & \pm 4.14 & \pm 2.42 \\
\hline
\end{tabular}

By examining Tables 2 and 3, there are some interesting points can be generally found. Firstly, the statistic features were consistently outperforms the spectral feature in most of groups. It can be concluded that although the spectral features is potentially used in most of speech recognition, this feature is harder to be intuitively correlated with the affective state such in embolic signal recognition. In the meantime, the statistic feature is potentially improving embolic identification accuracy over spectral feature. Moreover, the combination of MEBR, PEBR, entropy, standard deviation and maximum peak yields even further improvement in most cases.

Secondly, it was found the FkNCN classifier to be more intuitive than other tested classifier in all groups. As an extension of the $\mathrm{kNCN}$ and FkNN classifiers, the behavior of FkNCN was similar to these classifiers. Nevertheless, the FkNCN employed more information in training samples distribution and weighting distance among $\mathrm{k}$ nearest centroid neighbors. As a result, the FkNCN classifier was able to correctly classify the samples as it provided more information than the other classifiers in term of samples distribution and weighting distance.

\section{Conclusions}

In this paper, the embolic identification system based on ultrasound signal analysis has been studied and successfully implemented. The ultrasound signal data were obtained from five independent data sets of patients' group with different probe position and transmitted frequency varies from $1 \mathrm{MHz}$ to $3 \mathrm{MHz}$. subsequently, the data were extracted in the spectral feature i.e. LPC and statistical feature i.e. the combination of MEBR, PEBR, entropy, standard deviation and maximum peak. The segmented data then were compared with four classifiers from $\mathrm{NN}$ family i.e. $\mathrm{kNN}$, FkNN, kNCN and FkNCN. A series of experiments, based on different features and classifiers were employed and the results indicate that statistical features outperform the spectral feature with more than $80 \%$ were obtained in all tested groups. The results also observed that the FkNCN provide the best classification among the four classifiers with more than $78 \%$ were obtained by using the spectral feature and more than $88 \%$ by using the statistical feature.

\section{Acknowledgment}

The authors would like to thank the financial support provided by Fundamental Research Grant of Ministry of Education of Malaysia, 203/PELECT/6071266 (USM) and Professor Dr. Jafri Malim Abdullah from Hospital Universiti Sains Malaysia for all forms of assistance during this research work.

\section{References}

[1] L. M. Brass. Stroke. In: Yale University School of Medicine Heart Book.

[2] E. Bernd Ringelstein, D. W. Droste, V. L. Babikian, "Consensus on Microembolus Detection by TCD", Stroke, 1998, vol 29(3), pp. 725729. doi:10.1161/01.STR.29.3.725.

[3] S. Neurol, "Transcranial Doppler Ultrasound: Technique and Application", 2014, doi:10.1055/s-0032-1331812.

[4] N. Aydin, F. Marvasti, H. S. Markus, "Embolic Doppler Ultrasound Signal Detection Using Discrete Wavelet Transform", IEEE Trans Inf Technol Biomed, 2004, vol 8(2), pp. 182-190.

[5] G. A. Darbellay, R. Duff, J. M. Vesin, Solid or gaseous circulating brain emboli: are they separable by transcranial ultrasound? J Cereb Blood Flow Metab, 2004, vol 24(8), pp. 860-868, doi:10.1097/01.WCB.0000126235.54306.FA.

[6] N. Benoudjit, K. Ferroudji, M. Bahaz, A. Bouakaz, "In vitro microemboli classification using neural network models and RF signals", Ultrasonics. 2011, vol 51(3), pp. 247-252. doi:10.1016/j.ultras.2010.09.002.

[7] K. Ferroudji, N. Benoudjit, M. Bahaz, A. Bouakaz, U. M. R. Inserm, U. Cnrs, "Feature Selection Based on RF Signals and KNN Rule: Application to Microemboli Classification", 7th Int Work Syst Signal Process their Appl, 2011, pp. 251-254.

[8] K. Ferroudji, M. Bahaz, N. Benoudjit, A. Bouakaz, "Microemboli Classification using Non-linear Kernel Support Vector Machines and RF signals', Rev des Sci la Technol, 2012, pp. 3:79-87.

[9] S.G. Ballakur, "The Detection of Microemboli in Blood using Doppler Ultrasound: Signal Analysis and Detector Performance", December, 2007.

[10] B. R. Bollinger, "Dual-Frequency Ultrasoud Detection and Sizing of Microbubbles for Studying Decompression Sickness", 2008.

[11] E. Fix, J. Hodges, "Discriminatory analysis, nonparametric discrimination: consistency properties", Technical Report 4, USAF School of Aviation Medicine, Randolph Field, Texas, 1951.

[12] J. M. Keller, M. R. Gray, J. A. Givens, "A fuzzy k-nearest neighbor algorithm", IEEE Transactions on Systems, Man and Cybernetics, 1985, vol 4, pp. 580-585.

[13] J. S. Sanchez, F. Pla, F. J. Ferri, "On the use of neighbourhood-based non- parametric classifiers", Pattern Recognition Letters, 1997, vol 18 , pp. $1179-1186$

[14] L. R. Rabiner, R. W. Schafer, "Digital signal processing of speech signal", New Jersey, Prentice Hall Inc, 1978.

[15] B. A. Rosdi, H. Jaafar, D. A. Ramli. "Finger vein identification using fuzzy-based k-nearest centroid neighbor classifier" In: THE 2ND ISM INTERNATIONAL STATISTICAL CONFERENCE 2014 (ISM-II): Empowering the Applications of Statistical and Mathematical Sciences, 2015, pp. 649-654. 